\title{
EXISTENCE OF CRITICAL POINTS FOR SOME NONCOERCIVE FUNCTIONALS
}

\author{
David ARCOYA ${ }^{\text {a }}$, Lucio BOCCARDO ${ }^{\text {b }}$, Luigi ORSINA ${ }^{\text {b }}$ \\ ${ }^{a}$ Departamento de Análisis Matemático, Universidad de Granada, 18071-Granada, Spain \\ b Dipartimento di Matematica "Guido Castelnuovo", Università degli Studi di Roma, Piazzale A. Moro 5, \\ 00185, Roma, Italy
}

Received 10 April 2000

ABSTRACT. - In this paper we study critical points problems for some integral functionals with principal part having degenerate coerciveness, whose model is

$$
J(v)=\frac{1}{2} \int_{\Omega} \frac{|\nabla v|^{2}}{(b(x)+|v|)^{2 \alpha}}-\frac{1}{m} \int_{\Omega}|v|^{m}, \quad v \in H_{0}^{1}(\Omega),
$$

with $1<m<2^{*}(1-\alpha)$. We will prove several existence and nonexistence results depending on different assumptions on both $m$ and $\alpha$.

(C) 2001 L'Association Publications de l'Institut Henri Poincaré. Published by Elsevier B.V. All rights reserved

RÉSUMÉ. - Dans cet article nous étudions les points critiques de certaines fonctionnelles intégrales dont la coercitivité de la partie pricipale est dégénérée dont le modele est

$$
J(v)=\frac{1}{2} \int_{\Omega} \frac{|\nabla v|^{2}}{(b(x)+|v|)^{2 \alpha}}-\frac{1}{m} \int_{\Omega}|v|^{m}, \quad v \in H_{0}^{1}(\Omega),
$$

avec $1<m<2 *(1-\alpha)$. Nous prouvons plusieurs résultats d'existence et de non existence suivant les hypotheses sur $m$ et $\alpha$.

(C) 2001 L'Association Publications de l'Institut Henri Poincaré. Published by Elsevier B.V. All rights reserved

\section{Introduction}

For a bounded domain $\Omega \subset \mathbb{R}^{N}(N>2)$, minimization problems in the Sobolev space $H_{0}^{1}(\Omega)$ for integral functionals whose principal part depends on $x, v$ and $\nabla v$ as

$$
\frac{1}{2} \int_{\Omega} a(x, v)|\nabla v|^{2}
$$

with $a(x, s) \geqslant \alpha_{0}>0,(x \in \Omega, s \in \mathbb{R})$, are now classic.

E-mail addresses: boccardo@mat.uniroma.it (L. Boccardo), orsina@mat.uniroma1.it (L. Orsina). 
Conversely, the study of critical points is quite more recent (see $[2,3,6])$. In this framework, the main difficulties are that the functional is not differentiable on the whole $H_{0}^{1}(\Omega)$, but only in $H_{0}^{1}(\Omega) \cap L^{\infty}(\Omega)$, even if $a(x, s)$ is smooth, and that the associated differential operator, that is

$$
-\operatorname{div}(a(x, v) \nabla v)+\frac{1}{2} \frac{\partial a}{\partial s}(x, v)|\nabla v|^{2},
$$

involves a lower order term with quadratic growth in the gradient, which may not be in the dual space $H^{-1}(\Omega)$.

Recently (see [5]), minimization results for integral functionals whose principal part is

$$
\frac{1}{2} \int_{\Omega} a(x, v)|\nabla v|^{2}
$$

under the weak assumption $a(x, s)>0$ (that is, of degenerate coerciveness), were proved. Specifically, the authors considered functionals like

$$
\frac{1}{2} \int_{\Omega} \frac{|\nabla v|^{2}}{(b(x)+|v|)^{2 \alpha}}-\int_{\Omega} h v, \quad v \in H_{0}^{1}(\Omega),
$$

where $\alpha \in(0,1 / 2), 0<\beta_{1} \leqslant b(x) \leqslant \beta_{2}$ and $h \in H^{-1}(\Omega)$.

In this paper we shall study critical points problems for some integral functionals with principal part having degenerate coerciveness, whose model is

$$
J(v)=\frac{1}{2} \int_{\Omega} \frac{|\nabla v|^{2}}{(b(x)+|v|)^{2 \alpha}}-\frac{1}{m} \int_{\Omega}|v|^{m}, \quad v \in H_{0}^{1}(\Omega),
$$

with $1<m<2^{*}(1-\alpha)$. The derivative of $J$ is given by

$$
\left\langle J^{\prime}(u), v\right\rangle=\int_{\Omega} \frac{\nabla u \nabla v}{(b(x)+|u|)^{2 \alpha}}-\alpha \int_{\Omega} \frac{|\nabla u|^{2} u v}{(b(x)+|u|)^{1+2 \alpha}|u|}-\int_{\Omega}|u|^{m-2} u v,
$$

for every $v \in H_{0}^{1}(\Omega) \cap L^{\infty}(\Omega)$.

This explains our concept of critical point of $J$, that is a function $u \in H_{0}^{1}(\Omega) \cap L^{\infty}(\Omega)$ satisfying $\left\langle J^{\prime}(u), v\right\rangle=0$ for every $v \in H_{0}^{1}(\Omega) \cap L^{\infty}(\Omega)$. In this way, we can see the critical points of $J$ as solutions of the boundary value problem

$$
\begin{gathered}
-\operatorname{div}\left(\frac{\nabla u}{(b(x)+|u|)^{2 \alpha}}\right)-\alpha \frac{|\nabla u|^{2}}{(b(x)+|u|)^{1+2 \alpha}} \frac{u}{|u|}=|u|^{m-2} u, \quad x \in \Omega, \\
u \in H_{0}^{1}(\Omega) \cap L^{\infty}(\Omega) .
\end{gathered}
$$


Remark that, even if the function $\frac{|\xi|^{2}}{(b(x)+|s|)^{1+2 \alpha}} \frac{s}{|s|}$ is not continuous for $s=0$, the term

$$
\frac{|\nabla u|^{2}}{(b(x)+|u|)^{1+2 \alpha}} \frac{u}{|u|}
$$

appearing in the Euler equation of $J$ is well defined and measurable since where $u=0$ we have $\nabla u=0$ almost everywhere (Stampacchia Theorem).

Let us state the precise assumptions on the functional $J$ that we will study below. Let $\Omega$ be a bounded, open subset of $\mathbb{R}^{N}, N>2$.

Let $a: \Omega \times \mathbb{R} \rightarrow \mathbb{R}$ be a Carathéodory function (that is, measurable with respect to $x$ in $\Omega$ for every $s$ in $\mathbb{R}$, and continuous with respect to $s$ in $\mathbb{R}$ for almost every $x$ in $\Omega$ ) such that

$$
\frac{c_{1}}{(1+|s|)^{2 \alpha}} \leqslant a(x, s) \leqslant c_{2},
$$

for almost every $x$ in $\Omega$, for every $s$ in $\mathbb{R}$, where $c_{1}$ and $c_{2}$ are positive constants, and

$$
0 \leqslant \alpha<\frac{N}{2 N-2}
$$

(note that $\frac{N}{2 N-2} \in\left(\frac{1}{2}, 1\right)$ for every $N>2$ ). We also assume that the function $s \mapsto a(x, s)$ is differentiable on $\mathbb{R}$ for almost every $x$ in $\Omega$, and its derivative $a_{s}(x, s) \equiv \frac{\partial a}{\partial s}(x, s)$ is such that

$$
-2 \beta a(x, s) \leqslant a_{s}(x, s)(1+|s|) \operatorname{sgn}(s) \leqslant 0,
$$

for almost every $x$ in $\Omega$, for every $|s| \geqslant s_{0}$, where $\beta$ is a positive constant such that

$$
0<\beta<c_{1}
$$

and $s_{0}>0$.

As examples of functions $a$ satisfying assumptions (1.1) and (1.3) we can consider either

$$
a(x, s)=\frac{1}{(b(x)+|s|)^{2 \alpha}},
$$

with $0<\beta_{1} \leqslant b(x) \leqslant \beta_{2}$, or

$$
a(x, s)=\frac{1}{\left(1+s^{2}\right)^{\alpha}} .
$$

Let $F: \Omega \times \mathbb{R} \rightarrow \mathbb{R}$ be a Carathéodory function satisfying the following assumption:

$$
|F(x, s)| \leqslant \frac{K_{1}}{m}|s|^{m}+K_{2},
$$

for almost every $x$ in $\Omega$, for every $s$ in $\mathbb{R}$, where $K_{1}, K_{2}$ are positive constants, and defining $2^{*}=2 N /(N-2)$, the Sobolev embedding exponent,

$$
1<m<2^{*} .
$$


In some of our results we need a stronger condition on $F$ than (1.5); namely, we assume that the derivative $f(x, s)=\frac{\partial F}{\partial s}(x, s)$ satisfies

$$
|f(x, s)| \leqslant K_{1}^{\prime}|s|^{m-1}+K_{2}^{\prime},
$$

for almost every $x$ in $\Omega$ and for every $s$ in $\mathbb{R}$, where $K_{1}^{\prime}, K_{2}^{\prime}$ are positive constants.

We define, for $v$ in $H_{0}^{1}(\Omega)$, the functional

$$
J(v)=\frac{1}{2} \int_{\Omega} a(x, v)|\nabla v|^{2}-\int_{\Omega} F(x, v) .
$$

Observe that by assumption (1.1)-(1.2) and (1.5)-(1.6), $J$ is well defined on $H_{0}^{1}(\Omega)$. Furthermore, by the assumptions on $a$ and $F, J$ is also differentiable along directions in $H_{0}^{1}(\Omega) \cap L^{\infty}(\Omega)$, and its derivative is given by

$$
\left\langle J^{\prime}(v), w\right\rangle=\int_{\Omega} a(x, v) \nabla v \cdot \nabla w+\int_{\Omega} a_{s}(x, v)|\nabla v|^{2} w-\int_{\Omega} f(x, v) w,
$$

for every $v$ in $H_{0}^{1}(\Omega)$ and for every $w$ in $H_{0}^{1}(\Omega) \cap L^{\infty}(\Omega)$.

The behaviour of the functional $J$ may be different depending on the assumptions made on $m$. If $m$ is "small enough", then $J$ has a global minimum on $H_{0}^{1}(\Omega)$.

THEOREM 1.1. - Let us assume (1.1)-(1.2) and (1.5) with $1<m<2(1-\alpha)$. Suppose furthermore that

$$
\lim _{s \rightarrow 0} \frac{F(x, s)}{s^{2}}=+\infty, \quad \text { uniformly with respect to } x \text { in } \Omega,
$$

Then $J$ has a global nontrivial minimum $u$ in $H_{0}^{1}(\Omega) \cap L^{\infty}(\Omega)$.

On the other hand, if $m>2(1-\alpha)$ the functional is indefinite and global minimization is no longer possible. In the particular case $2<m<2^{*}(1-\alpha)$, and under some further assumptions on $F$ we will apply the version of the Mountain Pass Theorem [1] given in [2] for nondifferentiable functionals, to show the existence of a nontrivial critical point in $H_{0}^{1}(\Omega) \cap L^{\infty}(\Omega)$.

THEOREM 1.2. - Let us assume (1.1)-(1.4) and (1.7) with $2<m<2 *(1-\alpha)$. Suppose furthermore that $F$ satisfies

$$
\begin{gathered}
\lim _{s \rightarrow 0} \frac{F(x, s)}{s^{2}}=0, \quad \text { uniformly with respect to } x \text { in } \Omega, \\
\lim _{s \rightarrow+\infty} \frac{F(x, s)}{s^{2}}=+\infty, \quad \text { uniformly with respect to } x \text { in } \Omega,
\end{gathered}
$$

and that there exist $r>2$ and $s_{0}>0$ such that

$$
f(x, s) s \geqslant r F(x, s),
$$


for almost every $x$ in $\Omega$, for every $|s| \geqslant s_{0}$. Then $J$ has at least a nontrivial critical point $u$ belonging to $H_{0}^{1}(\Omega) \cap L^{\infty}(\Omega)$.

In order to prove Theorem 1.2, one of the main difficulties is to check that a suitable compactness condition of Palais-Smale type holds. Here the keystone in our approach will be the proof of the boundedness of the cluster points of the Palais-Smale sequences (see Remark 3.2). An additional difficulty also arises: the degenerate coerciveness in $H_{0}^{1}(\Omega)$ of the principal part of the differential operator, which will lead us to extend the functional to a larger space, namely $W_{0}^{1, q}(\Omega)$ for some $q<2$.

The case $2(1-\alpha)<m<\min \left\{2,2^{*}(1-\alpha)\right\}$ is also studied. In this case, however, we need to study the problem where the nonlinear term $F(x, s)$ is exchanged with $\lambda F(x, s)$ (with $\lambda>0$ ): also in this setting we prove the existence of a nontrivial local minimizer in $H_{0}^{1}(\Omega) \cap L^{\infty}(\Omega)$.

THEOREM 1.3. - Let $2(1-\alpha)<m<\min \left\{2^{*}(1-\alpha), 2\right\}$, and let a and $f$ satisfy assumptions (1.1)-(1.2), (1.7) and (1.9). Then there exists $\lambda_{0}>0$ such that the functional

$$
J_{\lambda}(v)=\frac{1}{2} \int_{\Omega} a(x, v)|\nabla v|^{2}-\lambda \int_{\Omega} F(x, v), \quad v \in H_{0}^{1}(\Omega),
$$

has at least one nontrivial critical point in $H_{0}^{1}(\Omega) \cap L^{\infty}(\Omega)$ for every $\lambda \in\left(0, \lambda_{0}\right)$.

Finally, by means of a change of variable in a model case, and using a result of [4], we will prove that it is possible to find a bounded solution of the Euler equation for $J_{\lambda}$ also in the case $2^{*}(1-\alpha)<m<2^{*}$, this time with $\lambda$ large. We will also deal with the case $m>2^{*}$ showing that, always in the model case, and in a starshaped domain, there are no positive solutions of the Euler equation for $J$. In order to do that, we will apply the now standard technique to show that the solutions of this equation satisfy a Pohožaev type inequality.

The plan of the paper is as follows: in the following Section we will prove Theorem 1.1, while Section 3 will be devoted to the proof of the main result of this paper, Theorem 1.2. Section 4 will study the problem for $J_{\lambda}$, while the final Section 5 will contain some remarks about a possible different approach to the study of $J$, as well as nonexistence of solutions for the Euler equation of $J$.

Notation. In the following we will use several times the following functions of a real variable, depending on a parameter $k>0$ :

$$
T_{k}(s)=\max (-k, \min (k, s)), \quad G_{k}(s)=s-T_{k}(s) .
$$

Furthermore, we will denote with $C$, or $C_{1}, C_{2}, \ldots$, various constant which may depend on the data of the problem, whose value may vary from line to line. If $1<q<N$, we will denote by $q^{*}$ the real number $N q /(N-q)$, the Sobolev embedding exponent for the space $W_{0}^{1, q}(\Omega)$. 


\section{Existence of a global minimum}

We begin with a technical result, which we will use several times in the following.

LEMMA 2.1. - If $\alpha \in(0, N /(2 N-2))$ and

$$
q=\frac{2 N(1-\alpha)}{N-2 \alpha}
$$

then for every measurable set $A \subset \Omega$, and for every $v \in H_{0}^{1}(\Omega)$ and $u \in W_{0}^{1, q}(\Omega)$, we have

$$
\int_{A}|\nabla v|^{q} \leqslant\left(\int_{A} \frac{|\nabla v|^{2}}{(1+|u|)^{2 \alpha}}\right)^{q / 2}\left(\int_{A}(1+|u|)^{q^{*}}\right)^{1-q / 2} .
$$

Proof. - If $v \in H_{0}^{1}(\Omega)$ and $u \in W_{0}^{1, q}(\Omega)$, we have from the Hölder inequality that

$$
\int_{A}|\nabla v|^{q}=\int_{A} \frac{|\nabla v|^{q}}{(1+|u|)^{\alpha q}}(1+|u|)^{\alpha q} \leqslant\left(\int_{A} \frac{|\nabla v|^{2}}{(1+|u|)^{2 \alpha}}\right)^{q / 2}\left(\int_{A}(1+|u|)^{\frac{2 \alpha q}{2-q}}\right)^{1-q / 2},
$$

for every measurable set $A \subset \Omega$. We conclude the proof by observing that, by (2.1), $q^{*}=2 \alpha q /(2-q)$.

Remark 2.2. - Observe that $q \in(1,2)$ if and only if $N>2$.

Proof of Theorem 1.1. - Let $q$ be as in the previous lemma. Reasoning as in [5], let us define the following functional on $W_{0}^{1, q}(\Omega)$ :

$$
\tilde{J}(v)= \begin{cases}\frac{1}{2} \int_{\Omega} a(x, v)|\nabla v|^{2}-\int_{\Omega} F(x, v), & \text { if } \int_{\Omega} a(x, v)|\nabla v|^{2}<+\infty, \\ +\infty, & \text { otherwise. }\end{cases}
$$

We are going to prove that $\tilde{J}$ is both coercive and weakly lower semicontinous on $W_{0}^{1, q}(\Omega)$ so that the existence of a minimum will follows from standard results. The weak lower semicontinuity is a consequence of a Theorem by De Giorgi (see [7]), and the complete proof of this fact can be found in [5]. As far as the coerciveness is concerned, it is enough to consider $v$ in $W_{0}^{1, q}(\Omega)$ such that $\tilde{J}(v)$ is finite. Reasoning as in Lemma 2.1 we obtain, also using Sobolev embedding,

$$
\int_{\Omega}|\nabla v|^{q} \leqslant C_{1}\left(\int_{\Omega} \frac{|\nabla v|^{2}}{(1+|v|)^{2 \alpha}}\right)^{q / 2}\left(1+\left(\int_{\Omega}|\nabla v|^{q}\right)^{q^{*} / q}\right)^{1-q / 2},
$$

which implies that if $R=\|v\|_{W_{0}^{1, q}(\Omega)}$, we have from (1.1)

$$
R^{q} \leqslant C_{2}\left(\int_{\Omega} a(x, v)|\nabla v|^{2}\right)^{q / 2}\left(1+R^{q^{*}}\right)^{1-q / 2}
$$


On the other hand, since $m<2(1-\alpha)<2^{*}(1-\alpha)=q^{*}$, one has

$$
\int_{\Omega}|v|^{m} \leqslant C_{3}\left(\int_{\Omega}|v|^{q^{*}}\right)^{m / q^{*}} \leqslant C_{4}\left(\int_{\Omega}|\nabla v|^{q}\right)^{m / q},
$$

that is

$$
\int_{\Omega}|v|^{m} \leqslant C_{4} R^{m}
$$

Thus, by (1.5) and (2.3) we obtain

$$
\tilde{J}(v) \geqslant C_{5} \frac{R^{2}}{\left(1+R^{q^{*}}\right)^{\frac{2}{q}-1}}-C_{6} R^{m}-C_{7} .
$$

Using the definition of $q$, it is easy to check that

$$
2-q^{*}\left(\frac{2}{q}-1\right)=2(1-\alpha)>m,
$$

so that

$$
\lim _{R \rightarrow+\infty} \tilde{J}(v)=+\infty
$$

that is, $\tilde{J}$ is coercive on $W_{0}^{1, q}(\Omega)$.

Let now $u$ be a minimum of $\tilde{J}$ on $W_{0}^{1, q}(\Omega)$. Let $\varphi_{1}$ be the first eigenfunction of the Laplacian in $\Omega$, which we suppose to have chosen with norm equal to one in $H_{0}^{1}(\Omega)$. Then, since $\varphi_{1}$ belongs to $H_{0}^{1}(\Omega)$,

$$
\tilde{J}\left(t \varphi_{1}\right)=J\left(t \varphi_{1}\right)=\frac{t^{2}}{2} \int_{\Omega} a\left(x, t \varphi_{1}\right)\left|\nabla \varphi_{1}\right|^{2}-\int_{\Omega} F\left(x, t \varphi_{1}\right) .
$$

Using assumption (1.9), it is easy to see that there exists $t>0$ such that $\tilde{J}\left(t \varphi_{1}\right)<0$, and so $u \neq 0$.

Furthermore, reasoning as in [5], it can be proved that $u$ belongs to $L^{\infty}(\Omega)$. This implies (by (1.1)) that $u$ also belongs to $H_{0}^{1}(\Omega)$, thus concluding the proof of the theorem.

Remark 2.3. - We remark explicitly that inequality (2.3) holds under assumptions (1.1)-(1.2), for every $v \in W_{0}^{1, q}(\Omega)$ such that $\int_{\Omega} a(x, v)|\nabla v|^{2}<+\infty$.

\section{Mountain Pass type critical points}

Our first result is the proof that sequences of Palais-Smale type for $J$ are convergent to critical points. 
LEMMA 3.1. - Let us assume (1.1)-(1.4), (1.7) with $2<m<2^{*}(1-\alpha)$, (1.12), and let $q$ as in Lemma 2.1. Then the functional $J$ satisfies the following compactness condition:

(C) Every sequence $\left\{u_{n}\right\} \subset H_{0}^{1}(\Omega) \cap L^{\infty}(\Omega)$ satisfying

$$
\lim _{n \rightarrow+\infty} J\left(u_{n}\right)=c,
$$

and, for some sequence $\left\{\varepsilon_{n}\right\} \subset(0, \infty)$ converging to zero,

$$
\left|\left\langle J^{\prime}\left(u_{n}\right), v\right\rangle\right| \leqslant \varepsilon_{n} \frac{\|v\|_{H_{0}^{1}(\Omega)}+\|v\|_{L^{\infty}(\Omega)}}{\left\|u_{n}\right\|_{H_{0}^{1}(\Omega)}+\left\|u_{n}\right\|_{L^{\infty}(\Omega)}}, \quad \forall v \in H_{0}^{1}(\Omega) \cap L^{\infty}(\Omega),
$$

possesses a subsequence which is weakly convergent in $W_{0}^{1, q}(\Omega)$ to some critical point $u \in H_{0}^{1}(\Omega) \cap L^{\infty}(\Omega)$ of $J$ with level $J(u)=c$.

Proof. - The proof is divided into 5 steps:

- Step 1: The sequence $\left\{u_{n}\right\}$ is bounded in $W_{0}^{1, q}(\Omega)$.

- Step 2: Up to a subsequence, $\left\{u_{n}\right\}$ weakly converges in $W_{0}^{1, q}(\Omega)$ to some $u \in$ $L^{\infty}(\Omega)$.

- Step 3: The function $u \in H_{0}^{1}(\Omega)$.

- Step 4: For fixed $k \geqslant\|u\|_{L^{\infty}(\Omega)}$, the following convergences hold:

$$
\begin{gathered}
\int_{\Omega} a\left(x, u_{n}\right)\left|\nabla G_{k}\left(u_{n}\right)\right|^{2} \rightarrow 0, \\
\int_{\Omega} a_{s}\left(x, u_{n}\right) v\left|\nabla G_{k}\left(u_{n}\right)\right|^{2} \rightarrow 0, \quad \forall v \in H_{0}^{1}(\Omega) \cap L^{\infty}(\Omega), \\
\left\|T_{k}\left(u_{n}\right)-u\right\|_{H_{0}^{1}(\Omega)} \rightarrow 0,
\end{gathered}
$$

as $n$ tends to infinity.

- Step 5: Conclusion: $u$ is a critical point of $J$.

Remark 3.2. - The main difference of our proof with respect to the standard proof of compactness conditions in theorems of Mountain Pass type is Step 2. Indeed, in the standard cases, the compactness condition is proved without the need of proving that the limit points (the critical points) are in $L^{\infty}(\Omega)$; their boundedness (as well as other regularity properties) is in general recovered from the equation they satisfy by standard bootstrap arguments. Conversely, in our case it is crucial to prove that the limit point is bounded in order to prove the compactness condition. Note also that the proof of Step 2 holds (in a much simpler way!) in the case $\alpha=0$ (i.e., in the "classical" case).

Proof of Step 1. - Taking $v=u_{n}$ in (3.2), we get

$$
\begin{aligned}
\varepsilon_{n} & \geqslant\left|\left\langle J^{\prime}\left(u_{n}\right), u_{n}\right\rangle\right| \\
& =\left.\left|\int_{\Omega} a\left(x, u_{n}\right)\right| \nabla u_{n}\right|^{2}+\frac{1}{2} \int_{\Omega} a_{s}\left(x, u_{n}\right) u_{n}\left|\nabla u_{n}\right|^{2}-\int_{\Omega} f\left(x, u_{n}\right) u_{n} \mid
\end{aligned}
$$




$$
=\left.\left|2 J\left(u_{n}\right)+\frac{1}{2} \int_{\Omega} a_{s}\left(x, u_{n}\right) u_{n}\right| \nabla u_{n}\right|^{2}+\int_{\Omega}\left[2 F\left(x, u_{n}\right)-f\left(x, u_{n}\right) u_{n}\right] \mid .
$$

Using (3.1), we thus have

$$
\lim _{n \rightarrow+\infty} \int_{\Omega}\left[f\left(x, u_{n}\right) u_{n}-2 F\left(x, u_{n}\right) u_{n}\right]-\frac{1}{2} \int_{\Omega} a_{s}\left(x, u_{n}\right) u_{n}\left|\nabla u_{n}\right|^{2}=2 c .
$$

Since $a_{s}(x, s) s \leqslant 0$ for $|s| \geqslant s_{0}$ by (1.3), and since, by assumption (1.12) one has

$$
f\left(x, u_{n}\right) u_{n}-2 F\left(x, u_{n}\right) \geqslant(r-2) F\left(x, u_{n}\right)-C_{1},
$$

we obtain from (3.6) that $\left\{F\left(x, u_{n}\right)\right\}$ is bounded in $L^{1}(\Omega)$ and thus, by (3.1), that there exists a positive constant $C_{2}$ such that

$$
\int_{\Omega} a\left(x, u_{n}\right)\left|\nabla u_{n}\right|^{2} \leqslant C_{2}, \quad \forall n \in \mathbb{N} .
$$

Now, by Lemma 2.1 (with $v=u=u_{n}, A=\Omega$ ) and the Sobolev embedding, if $q$ is as in (2.1),

$$
\int_{\Omega}\left|\nabla u_{n}\right|^{q} \leqslant C_{2}\left(\int_{\Omega}\left(1+\left|u_{n}\right|\right)^{q^{*}}\right)^{1-\frac{q}{2}} \leqslant C_{3}+C_{4}\left(\int_{\Omega}\left|\nabla u_{n}\right|^{q}\right)^{\frac{q^{*}}{q}\left(1-\frac{q}{2}\right)} .
$$

Observing that $\frac{q^{*}}{q}\left(1-\frac{q}{2}\right) \in(0,1)$, we obtain that $\left\{u_{n}\right\}$ is bounded in $W_{0}^{1, q}(\Omega)$.

Proof of Step 2. - Since $\left|G_{k}\left(u_{n}(x)\right)\right| \leqslant\left|u_{n}(x)\right|$ and $\nabla G_{k}\left(u_{n}(x)\right)=\nabla u_{n}(x)$ for almost every $x$ in $A_{k}^{n} \equiv\left\{\left|u_{n}\right| \geqslant k\right\}$, we deduce by taking $v=G_{k}\left(u_{n}\right)$ as test function in (3.2) that

$$
\begin{aligned}
& \int_{A_{k}^{n}} a\left(x, u_{n}\right)\left|\nabla G_{k}\left(u_{n}\right)\right|^{2}+\frac{1}{2} \int_{A_{k}^{n}} a_{s}\left(x, u_{n}\right) G_{k}\left(u_{n}\right)\left|\nabla G_{k}\left(u_{n}\right)\right|^{2} \\
& \quad \leqslant \int_{A_{k}^{n}} f\left(x, u_{n}\right) G_{k}\left(u_{n}\right)+\varepsilon_{n} .
\end{aligned}
$$

Observe now that, for $k \geqslant s_{0}$, and by (1.3), the derivative $a_{s}(x, s)$ is negative if $s \geqslant k$ and positive if $s \leqslant-k$. Hence, again by (1.3)

$$
a_{s}(x, s) G_{k}(s)=a_{s}(x, s)(s-k) \geqslant a_{s}(x, s)(s+1) \geqslant-2 \beta a(x, s), \quad \forall s \geqslant k,
$$

and

$$
a_{s}(x, s) G_{k}(s)=a_{s}(x, s)(s+k) \geqslant a_{s}(x, s)(s-1) \geqslant-2 \beta a(x, s), \quad \forall s \geqslant k \text {. }
$$

Thus,

$$
\frac{1}{2} a_{s}\left(x, u_{n}\right) G_{k}\left(u_{n}\right) \geqslant-\beta a\left(x, u_{n}\right)
$$


We obtain from (3.8) that

$$
(1-\beta) \int_{\Omega} a\left(x, u_{n}\right)\left|\nabla G_{k}\left(u_{n}\right)\right|^{2} \leqslant \int_{A_{k}^{n}} f\left(x, u_{n}\right) G_{k}\left(u_{n}\right)+\varepsilon .
$$

Thus, using (1.1) and (1.7), and the fact that $\left|G_{k}\left(u_{n}\right)\right| \leqslant\left|u_{n}\right|$,

$$
c_{1}(1-\beta) \int_{\Omega} \frac{\left|\nabla G_{k}\left(u_{n}\right)\right|^{2}}{\left(1+\left|u_{n}\right|\right)^{2 \alpha}} \leqslant C_{5} \int_{A_{k}^{n}}\left|u_{n}\right|^{m}+\varepsilon_{n} .
$$

By Lemma 2.1 (with $v=G_{k}\left(u_{n}\right), u=u_{n}$ and $A=A_{k}^{n}$ ) and since $k \geqslant 1$,

$$
\int_{\Omega}\left|\nabla G_{k}\left(u_{n}\right)\right|^{q} \leqslant C_{6}\left(\int_{A_{k}^{n}}\left|u_{n}\right|^{m}+\varepsilon_{n}\right)^{\frac{q}{2}}\left(\int_{A_{k}^{n}}\left|u_{n}\right|^{q^{*}}\right)^{1-\frac{q}{2}} .
$$

Now, Step 1 and the Hölder inequality (observe that $m<q^{*}$ ) imply that

$$
\begin{aligned}
\int_{\Omega}\left|\nabla G_{k}\left(u_{n}\right)\right|^{q} & \leqslant C_{6}\left(\varepsilon_{n}+\left(\int_{A_{k}^{n}}\left|u_{n}\right|^{q^{*}}\right)^{\frac{m}{q^{*}}}\left|A_{k}^{n}\right|^{1-\frac{m}{q^{*}}}\right)^{\frac{q}{2}}\left(\int_{A_{k}^{n}}\left|u_{n}\right|^{q^{*}}\right)^{1-\frac{q}{2}} \\
& \leqslant C_{7} \varepsilon_{n}^{q / 2}+\frac{2^{q^{*}}}{1-\alpha}\left(\int_{A_{k}^{n}}\left|u_{n}\right|^{q^{*}}\right)^{1-\frac{q}{2}\left(1-\frac{m}{q^{*}}\right)}\left|A_{k}^{n}\right|^{\frac{q}{2}\left(1-\frac{m}{q^{*}}\right)}
\end{aligned}
$$

Taking into account that

$$
\begin{aligned}
\int_{A_{k}^{n}}\left|u_{n}\right|^{q^{*}}=\int_{A_{k}^{n}}\left|u_{n}-k+k\right|^{q^{*}} & \leqslant C_{8} \int_{A_{k}^{n}}\left|G_{k}\left(u_{n}\right)\right|^{q^{*}}+C_{8} k^{q^{*}}\left|A_{k}^{n}\right| \\
& \leqslant C_{9}\left(\int_{A_{k}^{n}}\left|\nabla G_{k}\left(u_{n}\right)\right|^{q^{*}}\right)^{\frac{q^{*}}{q}}+C_{8} k^{q^{*}}\left|A_{k}^{n}\right|,
\end{aligned}
$$

we get

$$
\begin{aligned}
\int_{\Omega}\left|\nabla G_{k}\left(u_{n}\right)\right|^{q} \leqslant & \varepsilon_{n}^{\prime}+C_{10}\left(\int_{A_{k}^{n}}\left|\nabla G_{k}\left(u_{n}\right)\right|^{q}\right)^{\frac{q^{*}}{q}\left[1-\frac{q}{2}\left(1-\frac{m}{q^{*}}\right)\right]}\left|A_{k}^{n}\right|^{1-\frac{m}{q^{*}}} \\
& +C_{10} k^{q^{*}\left[1-\frac{q}{2}\left(1-\frac{m}{q^{*}}\right)\right]}\left|A_{k}^{n}\right| .
\end{aligned}
$$

Denoting $\theta=1-\frac{q}{2}\left(1-\frac{m}{q^{*}}\right)$ we have that $\theta \in(0,1)$, since $m<q^{*}=2^{*}(1-\alpha)$ and $N>2$. In addition, using $2(1-\alpha)<2<m$ we also get

$$
\theta \frac{q^{*}}{q}>1
$$

and thus 


$$
\begin{aligned}
\int_{\Omega}\left|\nabla G_{k}\left(u_{n}\right)\right|^{q} \leqslant & C_{10}\left(\int_{A_{k}^{n}}\left|\nabla G_{k}\left(u_{n}\right)\right|^{q}\right)^{\frac{\theta q^{*}}{q}-1}\left(\int_{A_{k}^{n}}\left|\nabla G_{k}\left(u_{n}\right)\right|^{q}\right)\left|A_{k}^{n}\right|^{1-\frac{m}{q^{*}}} \\
& +\varepsilon_{n}^{\prime}+C_{10} k^{\theta q^{*}}\left|A_{k}^{n}\right| .
\end{aligned}
$$

Since $\left\{u_{n}\right\}$ is bounded in $W_{0}^{1, q}(\Omega)$ there exists $k_{0} \in \mathbb{N}$ such that

$$
C_{10}\left(\int_{A_{k}^{n}}\left|\nabla G_{k}\left(u_{n}\right)\right|^{q}\right)^{\frac{\theta q^{*}}{q}-1}\left|A_{k}^{n}\right|^{1-\frac{m}{q^{*}}} \leqslant 1 / 2, \quad \forall k \geqslant k_{0} .
$$

Therefore, for $k \geqslant k_{0}$,

$$
\int_{\Omega}\left|\nabla G_{k}\left(u_{n}\right)\right|^{q} \leqslant \varepsilon_{n}^{\prime \prime}+C_{11} k^{\theta q^{*}}\left|A_{k}^{n}\right|
$$

Denote $K=\{k>0 /|\{u=k\}|=0\}$. Then, observe that letting $n$ tend to infinity, for every $k \in K,\left|A_{k}^{n}\right|$ converges to $\left|A_{k}\right|$ with $A_{k}=\{|u| \geqslant k\}$. Hence, since the norm in $W_{0}^{1, q}(\Omega)$ is weakly lower semicontinuous,

$$
\int_{\Omega}\left|\nabla G_{k}(u)\right|^{q} \leqslant C_{11} k^{\theta q^{*}}\left|A_{k}\right|, \quad \forall k \in K .
$$

Noting also that

$$
k^{q^{*}} \leqslant \frac{1}{\left|A_{k}\right|} \int_{A_{k}} k^{q^{*}} \leqslant \frac{1}{\left|A_{k}\right|} \int_{A_{k}}|u|^{q^{*}} \leqslant \frac{C_{12}}{\left|A_{k}\right|}, \quad \forall k \in K,
$$

we obtain

$$
\int_{\Omega}\left|\nabla G_{k}(u)\right|^{q} \leqslant C_{13} k^{q}\left|A_{k}\right|^{1-\theta+\frac{q}{q^{*}}}, \quad \forall k \in K .
$$

Since $|\mathbb{R} \backslash K|=0$, applying Lemma 5.2 of [9], we deduce that $u \in L^{\infty}(\Omega)$.

From now on we will restrict ourselves to the case $k \geqslant\|u\|_{L^{\infty}(\Omega)}$.

Proof of Step 3. - Remark that, by (1.1) and (3.7),

$$
\begin{aligned}
\int_{\Omega}\left|\nabla T_{k}\left(u_{n}\right)\right|^{2} & \leqslant(1+k)^{2 \alpha} \int_{\left\{\mid u_{n} \leqslant k\right\}} \frac{\left|\nabla T_{k}\left(u_{n}\right)\right|^{2}}{\left(1+\left|u_{n}\right|\right)^{2 \alpha}} \\
& \leqslant(1+k)^{2 \alpha} \int_{\Omega} \frac{\left|\nabla u_{n}\right|^{2}}{\left(1+\left|u_{n}\right|\right)^{2 \alpha}} \leqslant c(1+k)^{2 \alpha},
\end{aligned}
$$

which means that $\left\{T_{k}\left(u_{n}\right)\right\}$ is bounded in $H_{0}^{1}(\Omega)$. By this, it possesses a subsequence, still denoted by $\left\{T_{k}\left(u_{n}\right)\right\}$, converging to $T_{k}(u) \in H_{0}^{1}(\Omega)$ (as $n$ goes to infinity). Since 
$k \geqslant\|u\|_{L^{\infty}(\Omega)}$, we have that

$$
T_{k}(u)=u \in H_{0}^{1}(\Omega) .
$$

Proof of Step 4. - Using (1.7), we observe that, by the Rellich Theorem (recall that $\left.k \geqslant\|u\|_{L^{\infty}(\Omega)}\right)$,

$$
\lim _{n \rightarrow+\infty} \int_{\Omega}\left|u_{n}\right|^{m-1}\left|G_{k}\left(u_{n}\right)\right|=\int_{\Omega}|u|^{m-1}\left|G_{k}(u)\right|=0 .
$$

Thus, formula (3.3) is deduced from (3.9).

With respect to the proof of (3.4), note that by (1.3)

$$
\begin{aligned}
a_{s}\left(x, u_{n}\right) v & =a_{s}\left(x, u_{n}\right)\left(1+\left|u_{n}\right|\right) \operatorname{sgn}\left(u_{n}\right) \frac{v}{1+\left|u_{n}\right|} \operatorname{sgn}\left(u_{n}\right) \\
& \geqslant-2 \beta a\left(x, u_{n}\right) \frac{|v|}{1+\left|u_{n}\right|},
\end{aligned}
$$

and so

$$
\begin{aligned}
\int_{\Omega} a_{s}\left(x, u_{n}\right) v\left|\nabla G_{k}\left(u_{n}\right)\right|^{2} & \leqslant 2 \beta \int_{\Omega} a\left(x, u_{n}\right) \frac{|v|}{1+\left|u_{n}\right|}\left|\nabla G_{k}\left(u_{n}\right)\right|^{2} \\
& \leqslant 2 \beta \frac{\|v\|_{\infty}}{1+\left|u_{n}\right|} \int_{\left|u_{n}\right|>k} a\left(x, u_{n}\right)\left|\nabla G_{k}\left(u_{n}\right)\right|^{2},
\end{aligned}
$$

which, by (3.3), implies (3.4).

On the other hand, to prove (3.5) we consider the function $\varphi(s)=s \mathrm{e}^{\lambda s^{2}}$ with $\lambda>0$ chosen in such a way that

$$
\varphi^{\prime}(s)-4 \beta|\varphi(s)| \geqslant \frac{1}{2}, \quad \forall s \in \mathbb{R} .
$$

Define $v_{k}^{n}=\left(T_{k}\left(u_{n}\right)-u\right)$. Note that

$$
\begin{aligned}
\left\|\varphi\left(v_{k}^{n}\right)\right\|_{H_{0}^{1}(\Omega)}^{2} & =\int_{\Omega}\left|\nabla\left(T_{k}\left(u_{n}\right)-u\right)\right|^{2}\left[\varphi^{\prime}\left(T_{k}\left(u_{n}\right)-u\right)\right]^{2} \\
& \leqslant\left[\varphi^{\prime}(2 k)\right]^{2}\left[\left\|u_{n}\right\|_{H_{0}^{1}(\Omega)}^{2}+\left\|u_{n}\right\|_{L^{\infty}(\Omega)}^{2}\right],
\end{aligned}
$$

and

$$
\left\|\varphi\left(v_{k}^{n}\right)\right\|_{L^{\infty}(\Omega)} \leqslant \varphi(2 k) .
$$

Then, putting $v=\varphi\left(v_{k}^{n}\right)$ as test function in (3.2), we get

$$
\begin{aligned}
& \int_{\Omega} a\left(x, u_{n}\right) \nabla u_{n} \cdot \nabla v_{k}^{n} \varphi^{\prime}\left(T_{k}\left(u_{n}\right)-u\right) \\
& \quad+\frac{1}{2} \int_{\Omega} a_{s}\left(x, u_{n}\right) v_{k}^{n}\left|\nabla u_{n}\right|^{2} \varphi\left(v_{k}^{n}\right) \leqslant \varepsilon_{n}^{\prime}+\int_{\Omega} f\left(x, u_{n}\right) \varphi\left(v_{k}^{n}\right),
\end{aligned}
$$


with $\varepsilon_{n}^{\prime}$ tending to zero. Now observe that, by Rellich Theorem, and since $\varphi\left(v_{k}^{n}\right)$ converges to $\varphi(0)=0$,

$$
\lim _{n \rightarrow+\infty} \int_{\Omega} f\left(x, u_{n}\right) \varphi\left(v_{k}^{n}\right)=0 .
$$

Using also that $\nabla u_{n}=\nabla T_{k}\left(u_{n}\right)+\nabla G_{k}\left(u_{n}\right)$, we have

$$
\begin{aligned}
& \int_{\Omega} a\left(x, u_{n}\right) \nabla u_{n} \cdot \nabla v_{k}^{n} \varphi^{\prime}\left(T_{k}\left(u_{n}\right)-u\right) \\
& =\int_{\Omega} a\left(x, u_{n}\right) \nabla T_{k}\left(u_{n}\right) \cdot \nabla v_{k}^{n} \varphi^{\prime}\left(T_{k}\left(u_{n}\right)-u\right) \\
& \quad-\int_{\Omega} a\left(x, u_{n}\right) \nabla G_{k}\left(u_{n}\right) \cdot \nabla v_{k}^{n} \varphi^{\prime}\left(T_{k}\left(u_{n}\right)-u\right) \\
& =\int_{\left\{\left|u_{n}\right| \leqslant k\right\}} a\left(x, u_{n}\right) \nabla\left(T_{k}\left(u_{n}\right)-u\right) \cdot \nabla\left(T_{k}\left(u_{n}\right)-u\right) \varphi^{\prime}\left(T_{k}\left(u_{n}\right)-u\right) \\
& \quad+\int_{\left\{\left|u_{n}\right| \leqslant k\right\}} a\left(x, u_{n}\right) \nabla u \cdot \nabla v_{k}^{n} \varphi^{\prime}\left(T_{k}\left(u_{n}\right)-u\right) \\
& \quad-\int_{\Omega} a\left(x, u_{n}\right) \nabla G_{k}\left(u_{n}\right) \cdot \nabla u \varphi^{\prime}\left(T_{k}\left(u_{n}\right)-u\right) .
\end{aligned}
$$

In virtue of (1.1), $a\left(x, u_{n}\right)$ is bounded in $L^{\infty}(\Omega)$ and thus $a\left(x, u_{n}\right) \nabla u$ strongly converges to $a(x, u) \nabla u$ in $L^{2}\left(\Omega, \mathbb{R}^{N}\right)$, which together to the weak convergence of $\left\{T_{k}\left(u_{n}\right)\right\}_{n \in \mathbb{N}}$ to $u$ in $H_{0}^{1}(\Omega)$ and the $L^{\infty}(\Omega)$ weak* and almost everywhere convergence of $\varphi^{\prime}\left(T_{k}\left(u_{n}\right)-u_{n}\right)$ to $\varphi^{\prime}(0)=1$, yields

$$
\lim _{n \rightarrow+\infty} \int_{\left\{\left|u_{n}\right| \leqslant k\right\}} a\left(x, u_{n}\right) \nabla u \cdot\left(\nabla T_{k}\left(u_{n}\right)-u\right) \varphi^{\prime}\left(T_{k}\left(u_{n}\right)-u\right)=0 .
$$

Moreover, since

$$
\begin{aligned}
& \left|\int_{\Omega} a\left(x, u_{n}\right) \nabla G_{k}\left(u_{n}\right) \cdot \nabla u \varphi^{\prime}\left(T_{k}\left(u_{n}\right)-u\right)\right| \\
& \quad \leqslant c \varphi^{\prime}(2 k)\left(\int_{\Omega} a\left(x, u_{n}\right)\left|\nabla G_{k}\left(u_{n}\right)\right|^{2}\right)^{1 / 2}\left(\int_{\Omega}|\nabla u|^{2}\right)^{1 / 2},
\end{aligned}
$$

(3.3) and (3.10) imply that

$$
\lim _{n \rightarrow+\infty} \int_{\Omega} a\left(x, u_{n}\right) \nabla G_{k}\left(u_{n}\right) \cdot \nabla u \varphi^{\prime}\left(T_{k}\left(u_{n}\right)-u\right)=0 .
$$

Hence 


$$
\begin{aligned}
& \lim _{n \rightarrow+\infty}\left[\int_{\Omega} a\left(x, u_{n}\right) \nabla u_{n} \cdot \nabla v_{k}^{n} \varphi^{\prime}\left(T_{k}\left(u_{n}\right)-u\right)\right. \\
& \left.\quad-\int_{\left|u_{n}\right| \leqslant k} a\left(x, u_{n}\right)\left|\nabla\left(T_{k}\left(u_{n}\right)-u\right)\right|^{2} \varphi^{\prime}\left(T_{k}\left(u_{n}-u\right)\right)\right]=0 .
\end{aligned}
$$

Recalling (1.3) and taking into account that $\varphi\left(v_{k}^{n}\right)=\varphi(0)=0$ if $\left|u_{n}\right| \leqslant k$, one has

$$
\begin{aligned}
a_{s}\left(x, u_{n}\right) \varphi\left(v_{k}^{n}\right) & =a_{s}\left(x, u_{n}\right)\left(1+\left|u_{n}\right|\right) \operatorname{sgn}\left(u_{n}\right) \frac{\varphi\left(v_{k}^{n}\right)}{\left(1+\left|u_{n}\right|\right)} \operatorname{sgn}\left(u_{n}\right) \\
& \geqslant-2 \beta a\left(x, u_{n}\right)\left|\frac{\left|\varphi\left(v_{k}^{n}\right)\right|}{\left(1+\left|u_{n}\right|\right)}\right|
\end{aligned}
$$

Thus,

$$
\begin{aligned}
& \left.\left|\int_{\Omega} a_{s}\left(x, u_{n}\right) \varphi\left(v_{k}^{n}\right)\right| \nabla u_{n}\right|^{2} \mid \\
& \quad \leqslant 2 \beta \int_{\Omega} a\left(x, u_{n}\right)\left|\nabla u_{n}\right|^{2}\left|\varphi\left(v_{k}^{n}\right)\right| \\
& \quad 2 \beta \int_{\left\{\left|u_{n}\right| \leqslant k\right\}} a\left(x, u_{n}\right)\left|\nabla T_{k}\left(u_{n}\right)\right|^{2}\left|\varphi\left(v_{k}^{n}\right)\right|+2 \beta \int_{\Omega} a\left(x, u_{n}\right)\left|\nabla G_{k}\left(u_{n}\right)\right|^{2}\left|\varphi\left(v_{k}^{n}\right)\right| \\
& \quad \leqslant 4 \beta \int_{\left\{\left|u_{n}\right| \leqslant k\right\}} a\left(x, u_{n}\right)\left|\nabla\left(T_{k}\left(u_{n}\right)-u\right)\right|^{2}\left|\varphi\left(v_{k}^{n}\right)\right|+4 \beta c_{2} \int_{\left\{\left|u_{n}\right| \leqslant k\right\}}|\nabla u|^{2}\left|\varphi\left(v_{k}^{n}\right)\right| \\
& \quad+2 \beta|\varphi(2 k)| \int_{\Omega} a\left(x, u_{n}\right)\left|\nabla G_{k}\left(u_{n}\right)\right|^{2},
\end{aligned}
$$

so that from $(3.3),(3.10)$, and the fact that $\varphi\left(v_{k}^{n}\right)$ tends to zero we get

$$
\lim _{n \rightarrow+\infty} \int_{\Omega} a_{s}\left(x, u_{n}\right)\left|\nabla u_{n}\right|^{2} \varphi\left(v_{k}^{n}\right)-4 \beta \int_{\left\{\left|u_{n}\right| \leqslant k\right\}} a\left(x, u_{n}\right)\left|\nabla u_{n}\right|^{2}\left|\varphi\left(v_{k}^{n}\right)\right|=0 .
$$

Therefore the above estimate and (3.13) and (3.14) allows to conclude from (3.12) that

$$
\lim _{n \rightarrow+\infty} \int_{\left\{\left|u_{n}\right| \leqslant k\right\}} a\left(x, u_{n}\right)\left|\nabla\left(T_{k}\left(u_{n}\right)-u\right)\right|^{2}\left[\varphi^{\prime}\left(v_{k}^{n}\right)-4 \beta\left|\varphi\left(v_{k}^{n}\right)\right|\right]=0,
$$

which implies by (3.11) and (1.1) that

$$
\lim _{n \rightarrow+\infty} \int_{\left\{\left|u_{n}\right| \leqslant k\right\}} \frac{\left|\nabla\left(T_{k}\left(u_{n}\right)-u\right)\right|^{2}}{\left(1+\left|u_{n}\right|\right)^{2 \alpha}}=0
$$

that is

$$
\lim _{n \rightarrow+\infty} \int_{\left\{\left|u_{n}\right| \leqslant k\right\}}\left|\nabla\left(T_{k}\left(u_{n}\right)-u\right)\right|^{2}=0
$$


Using the fact that $u$ belongs to $H_{0}^{1}(\Omega)$, we thus have

$$
\lim _{n \rightarrow+\infty}\left\|T_{k}\left(u_{n}\right)-u\right\|_{H_{0}^{1}(\Omega)}^{2}=\lim _{n \rightarrow+\infty} \int_{\left\{\left|u_{n}\right| \leqslant k\right\}}\left|\nabla\left(T_{k}\left(u_{n}\right)-u\right)\right|^{2}+\lim _{n \rightarrow+\infty} \int_{\left\{\left|u_{n}\right|>k\right\}}|\nabla u|^{2}=0,
$$

which proves (3.5).

Proof of Step 5. - The function $u$ is a critical point of $J$ with level $J(u)=c$.

In fact, by using that $\nabla u_{n}=\nabla T_{k}\left(u_{n}\right)+\nabla G_{k}\left(u_{n}\right)$, we have that

$$
\begin{aligned}
& \int_{\Omega} a\left(x, u_{n}\right) \nabla T_{k}\left(u_{n}\right) \cdot \nabla v-\int_{\Omega} a_{s}\left(x, u_{n}\right) v\left|\nabla T_{k}\left(u_{n}\right)\right|^{2}-\int_{\Omega} f\left(x, u_{n}\right) v \\
& =\left\langle J^{\prime}\left(u_{n}\right), v\right\rangle-\int_{\Omega} a\left(x, u_{n}\right) \nabla G_{k}\left(u_{n}\right) \cdot \nabla v-\int_{\Omega} a_{s}\left(x, u_{n}\right) v\left|\nabla G_{k}\left(u_{n}\right)\right|^{2},
\end{aligned}
$$

for every $v \in H_{0}^{1}(\Omega) \cap L^{\infty}(\Omega)$. By (3.2), (3.4) and (3.3) we then obtain that

$$
\left[\int_{\Omega} a\left(x, u_{n}\right) \nabla T_{k}\left(u_{n}\right) \cdot \nabla v-\int_{\Omega} a_{s}\left(x, u_{n}\right) v\left|\nabla T_{k}\left(u_{n}\right)\right|^{2}-\int_{\Omega} f\left(x, u_{n}\right) v\right] \rightarrow 0 .
$$

Since by (3.5) the limit can be explicitely computed, we deduce

$$
\int_{\Omega} a(x, u) \nabla u \cdot \nabla v-\int_{\Omega} a_{s}(x, u) v|\nabla u|^{2}-\int_{\Omega} f(x, u) v=0,
$$

for every $v \in H_{0}^{1}(\Omega) \cap L^{\infty}(\Omega)$. Moreover, a similar argument as that used in Step 4 and (3.1) imply that

$$
J(u)=\lim _{n \rightarrow+\infty} J\left(u_{n}\right)=c .
$$

Proof of Theorem 1.2. - We apply the version of the Mountain Pass Theorem [1] given in [2] (see also [3]). In order to do this, let $u$ be in $H_{0}^{1}(\Omega)$, and set $R=\|u\|_{W_{0}^{1, q}(\Omega)}$ where $q$ is as in Lemma 2.1. Then by (2.3) (see Remark 2.3) we have

$$
\int_{\Omega} a(x, u)|\nabla u|^{2} \geqslant \frac{C_{1} R^{2}}{\left(1+R^{q^{*}}\right)^{2 / q-1}} .
$$

On the other hand, using the growth condition (1.5) and (1.10), we observe that for every $\varepsilon>0$ there exists $K_{\varepsilon}>0$ such that $F(x, s) \leqslant \varepsilon s^{2}+K_{\varepsilon} s^{m}$ for every $s$ in $\mathbb{R}$. Therefore, we have

$$
J(u) \geqslant \frac{C_{1} R^{2}}{\left(1+R^{q^{*}}\right)^{2 / q-1}}-\frac{\varepsilon}{\lambda_{1}} R^{2}-C_{2} R^{m},
$$

from which, by choosing $\varepsilon$ sufficiently small and using that $m>2$ and $q^{*}=2^{*}(1-\alpha)>$ 2 , it is easily deduced the existence of $R \in(0,1)$ and $\delta>0$ such that

$$
J(u) \geqslant \delta>0=J(0),
$$

for every $u$ in $H_{0}^{1}(\Omega)$ such that $\|u\|_{W_{0}^{1, q}(\Omega)}=R$. 
In addition, from (1.1) and (1.11) it follows

$$
J\left(t \varphi_{1}\right) \leqslant t^{2}\left[c_{2} \int_{\Omega}\left|\nabla \varphi_{1}\right|^{2}-\int_{\Omega} \frac{F\left(x, t \varphi_{1}\right)}{t^{2}}\right]<0,
$$

provided that $t>0$ is large enough. Hence we can choose $t_{0}>R /\left\|\varphi_{1}\right\|_{W_{0}^{1, q}(\Omega)}$ such that

$$
J\left(t_{0} \varphi_{1}\right)<0
$$

Consider now the set

$$
\Gamma=\left\{\gamma \in C^{0}\left([0,1], H_{0}^{1}(\Omega) \cap L^{\infty}(\Omega)\right): \gamma(0)=0, \gamma(1)=t_{0} \varphi_{1}\right\} .
$$

Then, by the embedding of $H_{0}^{1}(\Omega)$ into $W_{0}^{1, q}(\Omega)$ and standard connectedness arguments, we get

$$
c \equiv \inf _{\gamma \in \Gamma} \max _{t \in[0,1]} J(\gamma(t)) \geqslant \delta>0=\min \left\{J(0), J\left(t_{0} \varphi_{1}\right)\right\} .
$$

Take now a sequence $\left\{\gamma_{n}\right\}$ of paths in $\Gamma$ such that

$$
c \leqslant \max _{t \in[0,1]} J\left(\gamma_{n}(t)\right) \leqslant c+\frac{1}{2 n}, \quad \forall n \in \mathbb{N} .
$$

For fixed $n \in \mathbb{N}$, consider

$$
M_{n}=\max _{t \in[0,1]}\left[\left\|\gamma_{n}(t)\right\|_{H_{0}^{1}(\Omega)}+\left\|\gamma_{n}(t)\right\|_{L^{\infty}(\Omega)}\right] \geqslant t_{0}\left(1+\left\|\varphi_{1}\right\|_{L^{\infty}(\Omega)}\right)
$$

and observe that $\|\mid \cdot\| \|_{n}=\left(\|\cdot\|_{H_{0}^{1}(\Omega)}+\|\cdot\|_{L^{\infty}(\Omega)}\right) / M_{n}$ is a norm in $H_{0}^{1}(\Omega) \cap L^{\infty}(\Omega)$ which is equivalent to $\|\cdot\|_{H_{0}^{1}(\Omega)}+\|\cdot\|_{L^{\infty}(\Omega)}$. Then, applying [2, Theorem 2.1], we deduce the existence of a path $\bar{\gamma}_{n} \in \Gamma$ and a function $u_{n}=\bar{\gamma}_{n}\left(t_{n}\right) \in \bar{\gamma}_{n}([0,1])$ satisfying

$$
\begin{gathered}
c \leqslant \max _{t \in[0,1]} J\left(\bar{\gamma}_{n}(t)\right) \leqslant \max _{t \in[0,1]} J\left(\gamma_{n}(t)\right) \leqslant c+\frac{1}{2 n}, \\
\max _{t \in[0,1]}\left|\left\|\bar{\gamma}_{n}(t)-\gamma_{n}(t) \mid\right\|_{n} \leqslant \sqrt{\frac{1}{n}},\right. \\
c-\frac{1}{n} \leqslant J\left(u_{n}\right) \leqslant c+\frac{1}{2 n}, \\
\left|\left\langle J^{\prime}\left(u_{n}\right), v\right\rangle\right| \leqslant \sqrt{\frac{1}{n}}\left\|v|\||_{n}, \quad \forall v \in H_{0}^{1}(\Omega) \cap L^{\infty}(\Omega),\right.
\end{gathered}
$$

and for $n \in \mathbb{N}$ large enough,

$$
\begin{aligned}
\left\|u_{n}\right\|_{H_{0}^{1}(\Omega)}+\left\|u_{n}\right\|_{L^{\infty}(\Omega)}= & \left\|\bar{\gamma}_{n}\left(t_{n}\right)\right\|_{H_{0}^{1}(\Omega)}+\left\|\bar{\gamma}_{n}\left(t_{n}\right)\right\|_{L^{\infty}(\Omega)} \\
\leqslant & \left\|\bar{\gamma}_{n}\left(t_{n}\right)-\gamma_{n}\left(t_{n}\right)\right\|_{H_{0}^{1}(\Omega)}+\left\|\bar{\gamma}_{n}\left(t_{n}\right)-\gamma_{n}\left(t_{n}\right)\right\|_{L^{\infty}(\Omega)} \\
& +\left\|\bar{\gamma}_{n}\left(t_{n}\right)\right\|_{H_{0}^{1}(\Omega)}+\left\|\gamma_{n}\left(t_{n}\right)\right\|_{L^{\infty}(\Omega)} \\
\leqslant & 2 M_{n} .
\end{aligned}
$$


Therefore,

$$
\left|\left\langle J^{\prime}\left(u_{n}\right), v\right\rangle\right| \leqslant \sqrt{\frac{2}{n}} \frac{\|v\|_{H_{0}^{1}(\Omega)}+\|v\|_{L^{\infty}(\Omega)}}{\left\|u_{n}\right\|_{H_{0}^{1}(\Omega)}+\left\|u_{n}\right\|_{L^{\infty}(\Omega)}}, \quad \forall v \in H_{0}^{1}(\Omega) \cap L^{\infty}(\Omega),
$$

From Lemma 3.1 it then follows the existence of a critical point $u \in H_{0}^{1}(\Omega) \cap L^{\infty}(\Omega)$ of $J$ with critical level $c>0$. Clearly, $u \neq \equiv 0$ and the proof is finished.

\section{Existence by local minimization}

In the sequel we will prove Theorem 1.3, that is, the existence of critical point for the functional $J_{\lambda}$ defined in $H_{0}^{1}(\Omega)$ by setting

$$
J_{\lambda}(u)=\int_{\Omega} a(x, u)|\nabla u|^{2}-\lambda \int_{\Omega} F(x, u)
$$

for every $u \in H_{0}^{1}(\Omega)$, with $\lambda$ small enough.

Proof of Theorem 1.3. - If $q$ is as in Lemma 2.1, we consider, as in the proof of Theorem 1.1, the extension $\tilde{J}_{\lambda}$ of $J_{\lambda}$ to $W_{0}^{1, q}(\Omega)$ defined by

$$
\tilde{J}_{\lambda}(u)= \begin{cases}\int_{\Omega} a(x, u)|\nabla u|^{2}-\lambda \int_{\Omega} F(x, u), & \text { if } \int_{\Omega} a(x, u)|\nabla u|^{2}<+\infty \\ +\infty, & \text { otherwise. }\end{cases}
$$

Recall that for $u \in W_{0}^{1, q}(\Omega)$ satisfying

$$
\int_{\Omega} a(x, u)|\nabla u|^{2}<+\infty
$$

we have from (2.3) that there exist $R, \delta_{0}>0$ such that

$$
\int_{\Omega} a(x, u)|\nabla u|^{2} \geqslant \frac{C_{1}\|u\|_{W_{0}^{1, q}(\Omega)}^{2}}{\left(1+\|u\|_{W_{0}^{1, q}(\Omega)}^{q^{*}}\right)^{\frac{2}{q}-1}} \geqslant 2 \delta_{0},
$$

provided that $\|u\|_{W_{0}^{1, q}(\Omega)}=R$.

On the other hand, since (1.7), condition (1.5) is fulfilled with $m<q^{*}$ and we also have that

$$
\int_{\Omega} F(x, u) \leqslant K_{1} \int_{\Omega}|u|^{m}+K_{2} \int_{\Omega}|u| \leqslant C_{2}\|u\|_{W_{0}^{1, q}(\Omega)}^{m} .
$$


Consequently, if $\lambda \in\left(0, \delta_{0} /\left(C_{2} R^{2}\right)\right)$, we deduce for such a $u$ with norm $\|u\|_{W_{0}^{1, q}(\Omega)}=R$, that

$$
+\infty>\tilde{J}_{\lambda}(u) \geqslant \frac{C_{1}\|u\|_{W_{0}^{1, q}(\Omega)}^{2}}{\left(1+\|u\|_{W_{0}^{1, q}(\Omega)}^{q^{*}}\right)^{\frac{2}{q}-1}}-\lambda C_{2}\|u\|_{W_{0}^{1, q}(\Omega)}^{2} \geqslant \delta_{0} .
$$

In addition, as in the proof of Theorem 1.2, using now (1.9) and since $\varphi_{1}$ belongs to $H_{0}^{1}(\Omega)$,

$$
\tilde{J}_{\lambda}\left(t \varphi_{1}\right)=J_{\lambda}\left(t \varphi_{1}\right)<0,
$$

for $t>0$ small enough. Hence, taking into account that $\tilde{J}_{\lambda}$ is weakly lower semicontinuous on $W_{0}^{1, q}(\Omega)$ (see again [5]) we deduce the existence of $u_{0} \in \bar{B}\left(0, R ; W_{0}^{1, q}(\Omega)\right.$ ) such that

$$
\tilde{J}_{\lambda}\left(u_{0}\right)=\min _{\|u\|_{W_{0}^{1, q}(\Omega)}=R} \tilde{J}_{\lambda}(u) \leqslant J_{\lambda}\left(t \varphi_{1}\right)<0=J_{\lambda}(0)=\tilde{J}_{\lambda}(0) .
$$

Thus $u \not \equiv 0$ and by (4.1) we also get that $\|u\|_{W_{0}^{1, q}(\Omega)}<R$.

We claim that $u_{0} \in H_{0}^{1}(\Omega) \cap L^{\infty}(\Omega)$. Indeed, for $k>0$, we have that $\left\|T_{k}\left(u_{0}\right)\right\|_{W_{0}^{1, q}(\Omega)} \leqslant$ $\left\|u_{0}\right\|_{W_{0}^{1, q}(\Omega)}<R$ and so, by (1.1),

$$
\frac{1}{2} \int_{\Omega} \frac{\left|\nabla T_{k}\left(u_{0}\right)\right|^{2}}{\left(1+\left|T_{k}\left(u_{0}\right)\right|\right)^{2 \alpha}}=\frac{1}{2} \int_{\Omega} \frac{\left|\nabla T_{k}\left(u_{0}\right)\right|^{2}}{\left(1+\left|u_{0}\right|\right)^{2 \alpha}} \leqslant \frac{1}{2} \int_{\Omega} \frac{\left|\nabla u_{0}\right|^{2}}{\left(1+\left|u_{0}\right|\right)^{2 \alpha}}<+\infty .
$$

Thus, we can test the minimality of $u_{0}$ with $T_{k}\left(u_{0}\right)$, obtaining the inequality $\tilde{J}_{\lambda}\left(u_{0}\right) \leqslant$ $\tilde{J}_{\lambda}\left(T_{k}\left(u_{0}\right)\right)$, which implies by $(1.7)$

$$
\begin{aligned}
C_{1} \int_{\left\{\left|u_{0}\right|>k\right\}} \frac{\left|\nabla u_{0}\right|^{2}}{\left(1+\left|u_{0}\right|\right)^{2 \alpha}} & \leqslant \int_{\left\{\left|u_{0}\right|>k\right\}} a\left(x, u_{0}\right)\left|\nabla u_{0}\right|^{2} \leqslant \lambda \int_{\left\{\left|u_{0}\right|>k\right\}}\left[F\left(x, u_{0}\right)-F(k)\right] \\
& \leqslant \lambda C \int_{\left\{\left|u_{0}\right|>k\right\}}\left|u_{0}\right|^{m-1}\left|G_{k}\left(u_{0}\right)\right| .
\end{aligned}
$$

By a similar argument to that of the Step 2 of Lemma 3.1 we have that $u_{0} \in L^{\infty}(\Omega)$. Moreover,

$$
\begin{aligned}
\int_{\Omega}\left|\nabla u_{0}\right|^{2} & =\left(1+\left\|u_{0}\right\|_{L^{\infty}(\Omega)}\right)^{2 \alpha} \int_{\Omega} \frac{\left|\nabla u_{0}\right|^{2}}{\left(1+\left\|u_{0}\right\|_{L^{\infty}(\Omega)}\right)^{2 \alpha}} \\
& \leqslant\left(1+\left\|u_{0}\right\|_{L^{\infty}(\Omega)}\right)^{2 \alpha} \int_{\Omega} \frac{\left|\nabla u_{0}\right|^{2}}{\left(1+\left|u_{0}\right|\right)^{2 \alpha}}<+\infty,
\end{aligned}
$$

so that $u_{0}$ also belongs to $H_{0}^{1}(\Omega)$. Finally, we conclude noting that the regularity of $u_{0}$ implies that it is a critical point of $J_{\lambda}$. 


\section{Change of variable and nonexistence}

In this section we will use a particular form of the functional $J$, and show how some of the results we have obtained in the previous sections can be recovered starting from standard, known results on functionals of the Calculus of Variations.

We will consider the following (model) functional

$$
J(u)=\frac{1}{2} \int_{\Omega} \frac{|\nabla u|^{2}}{(1+|u|)^{2 \alpha}}-\frac{1}{m} \int_{\Omega}|u|^{m} .
$$

We define

$$
\Phi(s)=\frac{(1+|s|)^{1-\alpha}-1}{1-\alpha} \operatorname{sgn}(s),
$$

and observe that

$$
|\nabla \Phi(u)|^{2}=\frac{|\nabla u|^{2}}{(1+|u|)^{2 \alpha}}
$$

so that the functional $J$ can be rewritten, defining $H(s)=\Phi^{-1}(s)$, and $v=\Phi(u)$ as

$$
I(v)=\frac{1}{2} \int_{\Omega}|\nabla v|^{2}-\frac{1}{m} \int_{\Omega}|H(v)|^{m} .
$$

The function $H$ can be explicitly written:

$$
H(s)=\left\{[(1-\alpha) s+1]^{\frac{1}{1-\alpha}}-1\right\} \operatorname{sgn}(s) .
$$

Since $H^{\prime}(0)=1, H(s)$ behaves like $s$ close to the origin, while it behaves like $s^{\frac{1}{1-\alpha}}$ at infinity. Thus, in order for $H(v)^{m}$ to be a subcritical nonlinearity, it is necessary that $m<2^{*}(1-\alpha)$, which is exactly one of the assumptions we made in Theorem 1.2. This means that the critical point we found by means of Mountain Pass techniques can be seen as the "counterpart" of the critical point for $I$ which can be found applying the standard Mountain Pass Theorem. It is also clear that if $m<2(1-\alpha)$, then the term $H(v)^{m}$ is subquadratic, so that existence of critical points of $I$ by minimization is easily obtained (in this case, the minima of $I$ correspond exactly to the minima of $J$ via the change of variable).

However, the fact that $H(v)^{m}$ behaves like $v^{m}$ close to the origin, allows us to find existence of critical points of $I$ also in the case $2^{*}(1-\alpha)<m<2^{*}$. Indeed, let us introduce a positive parameter $\lambda$ as follows:

$$
I_{\lambda}(v)=\frac{1}{2} \int_{\Omega}|\nabla v|^{2}-\frac{\lambda}{m} \int_{\Omega}|H(v)|^{m}, \quad v \in H_{0}^{1}(\Omega) .
$$

The function $H(v)$ then satisfies, for $v$ close to zero, the assumptions of Theorem 8 of [4]. Thus, for $\lambda$ large, there exists critical points of Mountain Pass type for $I_{\lambda}$, with norm in $L^{\infty}(\Omega)$ which tends to zero as $\lambda$ tends to infinity. 
What happens if $m \geqslant 2^{*}$ ? In this case, a slightly modified version of the Pohozaev technique (see [10]) applies, yielding the following result.

THEOREM 5.1. - Let $\Omega$ be a starshaped, smooth domain, and let $m \geqslant 2^{*}$. Then the problem

$$
\left.\begin{array}{ll}
-\operatorname{div}\left(\frac{\nabla u}{(1+u)^{2 \alpha}}\right)-\alpha \frac{|\nabla u|^{2}}{(1+u)^{1+2 \alpha}}=u^{m-1}, & \text { in } \Omega, \\
u \geqslant 0, & \text { in } \Omega, \\
u \in H_{0}^{1}(\Omega) \cap L^{\infty}(\Omega) \cap H^{2}(\Omega), &
\end{array}\right\}
$$

has no nontrivial solutions.

Remark 5.2. - Up to now, we have always considered solutions in $H_{0}^{1}(\Omega) \cap L^{\infty}(\Omega)$, so that the assumption of $u$ in $H^{2}(\Omega)$ may seem too strong. This is not the case, since by a result of [8] every solution of (5.1) belongs to $H^{2}(\Omega)$.

Proof. - Let $u$ be a nonnegative solution in $H_{0}^{1}(\Omega) \cap L^{\infty}(\Omega) \cap H^{2}(\Omega)$ of (5.1). Multiplying by $x \cdot \nabla u$, and integrating on $\Omega$ yields, after some integrations by parts, and throwing away the integral on the boundary of $\Omega$ which has the right sign since $\Omega$ is starshped,

$$
\frac{1}{2^{*}} \int_{\Omega} \frac{|\nabla u|^{2}}{(1+u)^{2 \alpha}} \leqslant \frac{1}{m} \int_{\Omega} u^{m} .
$$

On the other hand, choosing

$$
v=\frac{1+u-(1+u)^{\alpha}}{1-\alpha}
$$

as test function in the Euler equation (5.1) yields

$$
\int_{\Omega} \frac{|\nabla u|^{2}}{(1+u)^{2 \alpha}}=\int_{\Omega} \frac{\left[1+u-(1+u)^{\alpha}\right] u^{m-1}}{1-\alpha},
$$

so that we have

$$
\frac{1}{2^{*}(1-\alpha)} \int_{\Omega}\left[1+u-(1+u)^{\alpha}\right] u^{m-1} \leqslant \frac{1}{m} \int_{\Omega} u^{m} .
$$

To prove that $u \equiv 0$ and to conclude the proof, it is sufficient to show that the function

$$
\frac{1}{2 *(1-\alpha)}\left[1+s-(1+s)^{\alpha}\right] s^{m-1}-\frac{1}{m} s^{m},
$$

is positive on $\mathbb{R}^{+}$. Dividing by $s^{m-1}$, this is equivalent to the positiveness of

$$
G(s)=\frac{1}{2^{*}(1-\alpha)}\left[1+s-(1+s)^{\alpha}\right]-\frac{s}{m} .
$$


We have $G(0)=0$, and

$$
G^{\prime}(s)=\frac{1}{2^{*}(1-\alpha)}\left[1-\alpha(1+s)^{\alpha-1}\right]-\frac{1}{m} .
$$

Since $G^{\prime}(0)=\frac{1}{2^{*}}-\frac{1}{m}$, the assumption $m \geqslant 2^{*}$ yields $G^{\prime}(0) \geqslant 0$. Moreover,

$$
G^{\prime \prime}(s)=\frac{\alpha}{2^{*}}(1+s)^{\alpha-2}>0,
$$

so that $G^{\prime}(s)$ is increasing, hence positive. Thus $G$ is increasing, hence positive on $\mathbb{R}^{+}$ and the proof is concluded.

\section{REFERENCES}

[1] Ambrosetti A., Rabinowitz P.H., Dual variational methods in critical point theory and applications, J. Funct. Anal. 14 (1973) 349-381.

[2] Arcoya D., Boccardo L., Critical points for multiple integrals of calculus of variations, Arch. Rat. Mech. Anal. 134 (1996) 249-274.

[3] Arcoya D., Boccardo L., Some remarks on critical point theory for nondifferentiable functionals, NoDEA Nonlinear Differential Equations Appl. 6 (1999) 79-100.

[4] Arcoya D., Gamez J.L., Orsina L., Peral I., Local existence results for sub-super-critical elliptic problems. Comm. Appl. Anal., to appear.

[5] Boccardo L., Orsina L., Existence and regularity of minima for integral functionals noncoercive in the energy space, Ann. Scuola Norm. Sup. Pisa 25 (1997) 95-130.

[6] Degiovanni M., Marzocchi M., A critical point theory for nonsmooth functionals, Ann. Mat. Pura Appl. 167 (1994) 73-100.

[7] De Giorgi E., Teoremi di Semicontinuità nel Calcolo Delle Variazioni, Lecture Notes, Istituto Nazionale di Alta Matematica, Roma, 1968.

[8] Donato P., Giachetti D., Quasilinear elliptic equations with quadratic growth in unbounded domains, Nonlinear Anal. 10 (1986) 791-804.

[9] Ladyzenskaya O.A., Uralceva N.N., Equations aux Dérivées Partielles de Type Elliptique, Dunod, Paris, 1968.

[10] Pohožaev S.I., Eigenfunctions of $\Delta u+\lambda f(u)=0$, Soviet Math. Dokl. 6 (1965) 1408-1411. 